\title{
Preface to the Special Focus manuscripts
}

\author{
Michael Hesse $\cdot$ Stephen Blackmore
}

Published online: 18 April 2013

(C) Springer-Verlag Wien 2013

The IPC XIII/IOPC IX 2012, with the main theme "Palynology and Palaeobotany in the Century of the Environment", was held in August 23-30, 2012 in Tokyo, Japan.

The Symposium SS10, entitled "Exine development and pattern formation, unifying ultrastructural and genetic approaches" and organised by Stephen Blackmore, Nina Gabarayeva and Michael Hesse, took place on August 28. It aimed to bring together experts who have approached pollen and spore development using various ultrastructural, theoretical and genetic approaches to work towards the development of a more unified understanding of exine organisation. The symposium was dedicated to the memory of John Rowley (1926-2010) a remarkable palynologist who had contributed to almost every aspect of palynology and who made an especially important contribution to the fields of pollen and spore development and the substructural organisation of the exine. Given his long and distinguished contribution to the field, it was little surprise that John Rowley had been known by most of those attending the symposium, whether as presenters or in the audience.

Text passages combined and adapted from "Exine development and
pattern formation, unifying ultrastructural and genetic approaches".
Organizers: Stephen Blackmore, Nina Gabarayeva and Michael
Hesse. Accepted proposal for Symposium SS 10 of the joint meeting
of 13th International Palynological Congress and 9th International
Organisation of Palaeobotany Conference, August 23-30 2012 in
Tokyo, Japan. S Blackmore, Pollen and spores: Microscopic keys to
understanding the earth's biodiversity-Pl. Syst. Evol. 263: 3-12,
2007. Hesse et al. 2009. Pollen terminology. An illustrated Handbook,
Springer.

M. Hesse $(\bowtie) \cdot$ S. Blackmore

Vienna, Austria

e-mail: michael.hesse@univie.ac.at

\section{Why is palynology important?}

Given the environmental challenges that lie ahead we have much to learn by exploring all aspects of biodiversity. One astonishingly informative field of investigation is palynology, the study of the pollen grains and spores of plants. The science of palynology is founded entirely upon the extraordinary organisational diversity and resistance to decay of the exine. Not surprisingly therefore, there has always been great interest in understanding how the complex, elaborate and often taxon-specific patterns of exine organisation are developed and have evolved. There has also been a strong interest in the theoretical basis of pollen and spore symmetry control, number and placement of germination sites, and surface pattern formation. More recently, there have been dramatic advances in the molecular genetics of pollen development based on insights from the model plant, Arabidopsis.

These microscopic, self-contained biological units are surrounded by chemically resistant cell walls with distinctive structures and symmetry. Pollen grains and spores can provide insights into such fundamental questions as how and when plants first colonised the land or how the earth's vegetation has developed through geological time and on finer time scales. They provide phylogenetic evidence important in plant systematics and model systems for understanding plant development at the cellular level.

The pollen grain is the point of origin and the carrier for the male gametes (sperm cells). What makes pollen grains so unique? Pollen grains represent an extra generation in seed plants, the highly reduced male gametophyte (the enclosing sporoderm and the cellular content, consisting of two or three cells, and the pollen tube). Pollen grains are therefore not simply parts of a plant, like leaves or seeds, but are the haploid counterpart of the much larger diploid 
plant body "as we see it in nature". These tiny, haploid, male organisms can be highly variable and distinctive in form. Amongst the important variable parameters are the pollen shape and size, the number, type and position of apertures and the pollen wall with its extremely diverse structure and sculpture. The comparative pollen (and spore) morphology of these characters is at least as important in plant systematics as any other morphological characters of the diploid generation. However, their developmental origins remain incompletely understood, hence the need for this symposium.

Stephen Blackmore and Michael Hesse, Guest Editors

The Guest Editors are very happy that Plant Systematics and Evolution now gives the opportunity to publish a Special Focus for selected palynological contributions to the Symposium SS10. 